\title{
Delayed surgery leads to reduced elbow range of motion in children with supracondylar humeral fractures managed at a referral hospital in sub-Saharan Africa
}

\author{
Claude Kasereka Masumbuko ${ }^{1}$ Edward Gakuya Mutheke², Benjamin Mbindyo ${ }^{2}$, Michael T Hawkes ${ }^{3}$
}

1. Université Catholique du Graben, Butembo, Democratic Republic of the Congo.

2. Kenyatta National Hospital, Nairobi, Kenya.

3. Department of Pediatrics, University of Alberta, Edmonton, Canada.

Emails: Kasereka Masumbuko Claude: claudemasumbuko@gmail.com; Gakuya Mutheke Edward: edward.gukaya@uonbi.ac.ke; Mbindyo Benjamin: mbindyo@uonbi.ac.ke

\begin{abstract}
Background: Supracondylar humeral fractures (SHFs) in children are associated with morbidity due to elbow stiffness. Timely operative management and/or physiotherapy are thought to reduce this complication, but pose challenges in settings with limited resources for health.

Methods: This prospective cohort study included 45 pediatric patients with isolated SHF at a large tertiary hospital in Nairobi, Kenya. Patients were managed non-operatively or operatively with varying wait times to surgery, with or without physiotherapy. The measurement of elbow ROM was done up to 12 weeks after removal of Kirshner wires and/or backslab.

Results: Elbow ROM increased in the follow-up period, yet residual restricted mobility in the flexion-extension plane was common. Delayed surgical management $\geq 7$ days was associated with reduced elbow ROM in the flexion-extension plane at 12 weeks median IQR $105^{\circ} 92^{\circ}-118^{\circ}$ vs $120^{\circ} 108^{\circ}-124^{\circ}, \mathrm{p}=0.029$. Physiotherapy was associated with reduced $\mathrm{ROM}$ at 12 weeks $\mathrm{p}=0.003$, possibly due to the use of prolonged immobilization.

Conclusion: In this study of pediatric SHFs at a resource-limited hospital, elbow flexion was restricted at 12 weeks follow-up and was associated with major delays in operative management. Quality of orthopedic surgical care and physiotherapy services in low-resource settings deserves further attention.

Keywords: Delayed surgery, reduced elbow range, supracondylar humeral fractures, sub-Saharan Africa.

DOI: https://dx.doi.org/10.4314/ahs.v19i3.32

Cite as: Masumbuko CK, Mutheke EG, Mbindyo B, Hawkes MT. Delayed surgery leads to reduced elbow range of motion in children with supracondylar humeral fractures managed at a referral hospital in sub-Saharan Africa. Afri Health Sci. 2019;19(3): $2565-2570$. bttps:/ / dx.doi.org/10.4314/abs.v19i3.32
\end{abstract}

\section{Introduction}

Supracondylar humerus fractures (SHFs) account for 13$17 \%$ of all paediatric fractures and $60-80 \%$ of all elbow injuries ${ }^{1}$. SHFs may be associated with morbidity; including malunion, neurovascular complications, compartment syndrome, and elbow stiffness ${ }^{2}$. Although several studies have documented excellent outcomes following surgical

\section{Corresponding author:}

Michael T Hawkes,

3-588 1140587 Ave

Edmonton, Alberta, Canada T6G 1C9

Tel: 780-248-5540, Fax: 1-888-790-1176

Email: mthawkes@ualberta.ca management of SHF in high-income countries ${ }^{3-7}$, few data are available from low- and middle-income countries where limited resources for health may impact quality of care and patient outcomes.

The timing of the treatment of SHFs in the acute setting has received significant attention. While some authors advocate for urgent operative management ${ }^{8}$, other studies in high-income settings have suggested that, in the absence of neurovascular compromise, surgery can safely be delayed by $8-21$ hours ${ }^{7,9,10}$. However, in settings with limited human resources for health and operating room capacity, delays of many days are not uncommon for surgical procedures ${ }^{11}$. Thus, the magnitude of delay in surgical management may be several-fold longer in resource-limited hospitals, the impact of which on patient

C) 2019 Masumbuko et al. Licensee African Health Sciences. This is an Open Access article distributed under the terms of the Creative commons Attribution License (https://creativecommons.org/licenses/BY/4.0), which permits unrestricted use, distribution, and reproduction in any medium, provided the original
} 
outcome has not been reported previously. The primary objective of this study was to evaluate the effect of delays in the surgical management of pediatric SHFs treatment on the range of motion ROM at the elbow at 12 weeks follow-up. As a secondary objective, we examined the effect of physiotherapy during the 12 week follow-up period on elbow ROM.

\section{Methods}

\section{Study setting}

This study was conducted at a large tertiary referral and teaching hospital in Kenya. Kenya ranks 145 of 186 on the United Nations Human Development Index United Nations, 2016121212 and has 0.14 physicians per 1,000 population compared with the US, where there are 2.3 physicians per 1,000 population World Bank, 20161313 13 Health expenditure based on gross domestic product is $5.7 \%$, which ranks the country $111^{\text {th }}$ of 189 overall World Bank, 20161313 13. Although the centre is a government-affiliated hospital, surgical procedures are primarily self-funded by the patient or their family.

\section{Study design}

This was a prospective observational study. Patients less than 15 years of age with radiologically confirmed isolated SHF were included. Patients were excluded if they intended to seek follow-up at another centre or if they had: open fracture; burn; bilateral supracondylar humerus fracture; neurovascular injury before treatment; multiple fractures; or ipsilateral forearm fractures.

The primary outcome measure was elbow ROM at 12 weeks follow-up, with reference to published normative data. Secondary outcomes included the longitudinal course of elbow ROM following management, and the effect of physiotherapy on elbow ROM.

A sample size calculation indicated that 34 patients were needed to detect a $15^{\circ} 10 \%$ difference in elbow flexion in patients with delayed $\geq 7$ days versus timely $<7$ days surgery with $80 \%$ power at $\alpha=0.05$ level of significance, using normative data for elbow flexion in boys age 2-8 years: mean $\mathrm{SD} 153^{\circ} 5^{\circ 14}$. We increased the sample size by $30 \%$ to account for potential loss to follow-up.

\section{Procedures}

Patients were identified in the casualty department, where written informed consent was obtained from the accom- panying parent or guardian. Demographic and clinical information was collected using a structured questionnaire. A lateral view radiograph centered at distal humerus, and a true anteroposterior view of the involved elbow were used to categorize patients according to Wilkin's modified Gartland classification. ${ }^{8}$

Non-displaced fractures were managed on a backslab cast and followed as out-patients. Patients with more severe injuries were admitted, pending surgical intervention. Surgical management consisted of closed reduction and pinning CRIF or open reduction and internal fixation with Kirshner wires ORIF. After reduction of the fracture, the injured elbow was placed in 90 degrees of flexion with the forearm in neutral position and secured in a back slab for 3 weeks. Patients were then discharged and managed as outpatients. Patients were reviewed at the orthopedic out-patients' clinic by the study team three weeks later for removal of pins and/or cast. Each patient was followed, from the date of cast and/or pin removal, every four weeks, up to 12 weeks. Affected elbow ROM was measured at each follow-up visit.

The measurements of elbow ROM were performed using standardized methods ${ }^{14}$. With the forearm in neutral position, the elbow was placed in angle at which the joint was immobilized. Using gentle active elbow ROM by encouraging the patient on motion as far as he could the limit of flexion, extension, forearm supination, and pronation were measured using a goniometer. To measure flexion and extension, the goniometer was centered at the distal humerus, which represent the approximate axis of elbow flexion-extension. The arms of the goniometer were then aligned parallel to the humerus and the forearm, respectively. To determine the extent of maximal forearm rotation, elbow ROM was performed by the researcher as previously described ${ }^{14}$.

Patients were encouraged but not required to attend physiotherapy session after cast and/or pin removal. At the end of the follow-up period, patients were asked if they had attended physiotherapy sessions or not. All patient were encouraged to perform daily elbow active ROM at home, as pain allowed.

\section{Statistical analysis}

To answer the primary objective, we examined the Spearman rank correlation $\varrho$ between the time interval from 
injury to intervention and the elbow ROM at 12 weeks. For secondary objectives, descriptive and comparative statistics were used as appropriate. Data processing was done using the statistical software SPSS 19 and GraphPad Prism.

\section{Ethics}

Institutional Research Ethics Committees approved this study. Written informed consent was obtained from parents or guardians of all patients.

\section{Results}

Forty-five children with SHFs were included, between October 2012 to February 2013. Demographic and clinical details are given in Table 1 . The median age was 7 years range 2-15 and 28/45 62\% were male. Most injuries occurred in the non-dominant limb. All patients with Gartland type I $n=6$ and II $n=10$ fractures were managed non-operatively and patients with Gartland type III fractures were managed by CRIF $n=1$ or ORIF $n=28$. The timing of non-operative management cast was within 1 day range 1-5 days of injury, compared to ORIF with a median delay of 7 days range 3-13 days.

\section{Table 1: Characteristics of patients, fractures, and management}

$\begin{array}{lc}\text { Variables } & \mathbf{n}=\mathbf{4 5}(\mathbf{\%}) \\ \text { Age [years], median (range) } & 7(2-15) \\ \text { Gender, n (\%) } & 17(38) \\ \text { Female } & 28(62) \\ \text { Male } & 13(29) \\ \text { Side of injury, n (\%) } & 32(71) \\ \text { Dominant limb } & \\ \text { Non-dominant limb } & 10(22) \\ \text { Severity (Gartland classification) } & 6(13) \\ \text { Type I } & 29(64) \\ \text { Type II } & 16(36) \\ \text { Type III } & 1(2.2) \\ \text { Management, n (\%) } & 28(62) \\ \text { Non-operative management } & 1(1-5) \\ \text { CRIF } & 1 * \\ \text { ORIF } & 7(3-13) \\ \text { Time from injury to treatment [days], median (range) } \\ \text { Non-operative management } \\ \text { CRIF } & 18(40) \\ \text { ORIF } & 27(60) \\ \text { Physiotherapy, n (\%) } & \\ \text { Yes } & \\ \text { No } & \end{array}$

\section{Rate of elbow ROM improvement following SHF} The change in the elbow ROM through multiple planes during recovery is shown in Figure 1. Table 2 shows the elbow ROM as a fraction of the expected normal range of motion based on published normative data ${ }^{14}$. Flexion was most markedly reduced, with a median IQR recovery of only $57 \% 41 \%-67 \%$ of the expected ROM by 12 weeks. Only one patient $2 \%$ achieved ROM in the flex- ion-extension plane within $15^{\circ}$ of expected. Better recovery in supination and pronation were observed Table 2 , with 44/45 98\%of patients achieving a ROM within $15^{\circ}$ of expected.

While the ROM flexion-extension improved from 4 weeks to 8 weeks, and from 8 weeks to 12 weeks, the incremental change was greater in the early interval [me- 
dian IQR $32^{\circ} 24^{\circ}-40^{\circ}$ ] than in the later interval [median IQR $\left.12^{\circ} 6^{\circ}-17^{\circ}, \mathrm{p}<0.0001\right]$. Similarly, the improvement in the ROM supination-pronation plane was greater from 4 to 8 weeks pins and or cast removal [median IQR $10^{\circ}$ $4^{\circ}-20^{\circ}$ ] than from 8 to 12 weeks post-treatment [median IQR $\left.0^{\circ} 0-4^{\circ}, \mathrm{p}<0.0001\right]$. A median IQR of $75 \% 67-83$ of gains in flexion-extension and 100\% 76-100 of gains in supination-pronation were achieved between week 4 to 8 of follow-up.

\section{Loss of ROM with delay in treatment of SHFs}

Delayed surgical management was associated with reduced range of motion at 12 weeks follow-up time postcast and pins removal. There was a significant inverse correlation between the time from injury to definitive management and the elbow ROM in the flexion-extension plane at 12 weeks $\varrho=-0.42, p=0.005$. Assuming a linear relationship between the variables, for each additional day of delayed surgery, there was a reduction in ROM flexion-extension at 12 weeks of $1.8^{\circ} 95 \% \mathrm{CI} 0.6^{\circ}$ to $3.0^{\circ}$; $\mathrm{p}=0.005$. The relationship between delay in management and reduced ROM was primarily among patients undergoing ORIF Figure $2, \varrho=-0.40, p=0.041$. Patients undergoing ORIF 7 or more days after injury had a flexion-extension elbow ROM of $105^{\circ} 92^{\circ}-118^{\circ}$, compared to $120^{\circ}$ $108^{\circ}-124^{\circ}$ among patients who were operated within 7 days $\mathrm{p}=0.029$. No significant relationship was observed between timing of non-operative management and outcome among patients with milder injuries.

\section{Effect of physiotherapy}

Among patients who underwent physiotherapy, the median IQR elbow flexion was $113^{\circ} 110^{\circ}-128^{\circ}$, compared to $129^{\circ} 120^{\circ}-137^{\circ}$ among patients who did not undergo physiotherapy $\mathrm{p}=0.003$. However, patients who underwent physiotherapy also had more severe injury at baseline $\mathrm{p}=0.032$. Stratifying the cohort by injury severity, we found no significant differences among patients with Gartl and type I or II fractures $\mathrm{p}=0.53$; however, patients with Gartland type III fractures had lower ROM at 12 weeks if they underwent physiotherapy [median IQR $99^{\circ}$ $90^{\circ}-116^{\circ}$ versus $\left.122^{\circ} 106^{\circ}-125^{\circ}, \mathrm{p}=0.001\right]$. Physiotherapists tended to employ an arm sling, resulting in moreprolonged immobilization: 39\% patients who underwent physiotherapy wore an arm sling for more than 4 weeks compared to $11 \%$ of patients who did not undergo physiotherapy $\mathrm{p}=0.028$. Moreover, use of an arm sling was associated with a lower range of motion in the flexion-extension plane at 12 weeks follow-up $p=0.008$. Thus, prolonged joint immobilization is an important confounder that may explain why physiotherapy was associated with restricted elbow ROM at 12 weeks.

\section{Discussion}

Our prospective study of outcomes of children with supracondylar fractures is distinct among other reports in the literature for its setting in a low- to middle-income country, with severe human resource and infrastructure limitations within the health care system. While other series from Africa have described the epidemiology of supracondylar fractures ${ }^{15,16}$ and challenges in their management at resource-constrained settings ${ }^{17}$, none have reported outcomes and determinants of poor recovery in such contexts. Characteristics of our cohort differ markedly from other reports in the medical literature from high-income settings: notably, delays in definitive surgical management of a week or more and relatively poor shortterm outcome.

In our cohort, only one patient $2 \%$ achieved ROM within $15^{\circ}$ of population norms in the flexion-extension plane by 12 weeks follow-up. In contrast, $79 \%$ to $97 \%$ of patients had ROM within $15^{\circ}$ of the contralateral uninjured elbow in other series ${ }^{3,4,12}$. These pronounced differences may be due to several factors. First, different measurement methods were used: comparison to published norms versus to the contralateral limb. Second, duration of follow-up was up to 12 weeks in our study, yet longer in others. Third, surgical tecnique differed: we used a posterior approach with triceps splitting whereas other authors used lateral or medial approaches. Finally, observed differences may be due to prolonged delays in management in our study compared to others.

Delays in the operative management of patients in our study are several-fold longer than other published series in high-income settings. The median delay in our study, 7 days, was more than 5 times longer than the average reported in these series. In contrast, in a study from the US, Gartl and type 3 fractures were managed within 19 hours range 0 to 48 hours $^{3}$. Another US series with delays on the order of days similar to our study involved only type 2 fractures that were managed with closed reduction ${ }^{13}$. Delayed surgical intervention may also be common at similar 
centres across the continent and in other resource-limited hospitals globally. For example, one study from Cameroon documented a mean delay of 7 days range 30 minutes to 120 days from initial consultation to definitive management of paediatric fractures. ${ }^{18}$ Increased attention to patient triage, effective use of operating room resources and manpower are potential solutions to alleviate the burden of morbidity identified in ours and previous studies.

Several case series from North America, Europe and China have evaluated whether delayed management of pediatric SHFs results in increased risk of complications s $^{3-7,9,10}$. Elbow ROM was examined in several of these series, and did not differ among children managed with early versus late intervention ${ }^{3,412}$. However, the magnitude of delay in these studies was on the order of half a day, in contrast to delays of a week or more in our study. Our study identified delay in surgical management as a significant determinant of restricted elbow ROM. Major differences in the time interval from injury to treatment between our study and series from higher income settings likely explain this discrepancy. Such extreme delays are outside the range of clinical practice in higher income settings and may plausibly lead to the sub-optimal outcomes we observed.

The role of physiotherapy after operative treatment of SHFs in children has been studied in a prospective randomized study which included 51 children with isolated SHFs type II or III who underwent ORIF and Kirshner wiring ${ }^{19}$. The authors found no significant difference in the final elbow ROM between patients who underwent physiotherapy and those who did not, although children receiving physiotherapy achieved a more rapid return of normal or near normal elbow ROM. These results contrast with our own, which suggested that patients undergoing physiotherapy have a restricted elbow ROM at 12 week follow-up. Several reasons for this discrepancy are possible: self-selection of more severely affected patients to seek physiotherapy although the association remained after adjustment for baseline fracture severity; variable and non-standardized physiotherapy regimens; variation between practitioners; variable time spent during each physiotherapy session, due in part to long wait-times and high patient volumes; frequency of the intervention one physiotherapy session per week, which was less than that recommended by other authors $2-3$ sessions per week ${ }^{19}$; and use of prolonged elbow joint immobilization. Although the findings of this study would need to be confirmed in other studies, reduced elbow ROM at 12 week follow-up among patients undergoing physiotherapy raises concerns about the quality of care of physiotherapy services in post-operative pediatric orthopedic care in the African hospital setting. Further studies are warranted to ensure physiotherapy interventions, as currently practiced in resource-limited settings, are not leading to harm.

In summary, pediatric SHFs were associated with residual restricted elbow ROM in the flexion-extension plane at 12 weeks follow-up. Delays in operative management were associated with reduced ROM. Physiotherapy was associated with restricted ROM, perhaps due to the use of prolonged immobilization. Relative to published studies, poor short-term outcomes in our study call attention to the need for more timely access to definitive surgical management, and a re-examination of the quality of physiotherapy services in the African hospital setting.

\section{Conflict of interest statement}

The authors have no conflict of interest to declare.

\section{References}

1. Allen SR, Hang JR, Hau RC. Review article: paediatric supracondylar humeral fractures: emergency assessment and management. Emerg Med Australas. 2010;225:418-26.

2. Battaglia TC, Armstrong DG, Schwend RM. Factors affecting forearm compartment pressures in children with supracondylar fractures of the humerus. J Pediatr Orthop. 2002;224:431-9.

3. Bales JG, Spencer HT, Wong MA, Fong YJ, Zionts LE, Silva M. The effects of surgical delay on the outcome of pediatric supracondylar humeral fractures. J Pediatr Orthop. 308:785-91.

4. Leet AI, Frisancho J, Ebramzadeh E. Delayed treatment of type 3 supracondylar humerus fractures in children. J Pediatr Orthop. 2002;222:203-7.

5. Gupta N, Kay RM, Leitch K, Femino JD, Tolo VT, Skaggs DL. Effect of surgical delay on perioperative complications and need for open reduction in supracondylar humerus fractures in children. J Pediatr Orthop. 2004;243:245-8.

6. Mehlman CT, Strub WM, Roy DR, Wall EJ, Crawford $\mathrm{AH}$. The effect of surgical timing on the perioperative complications of treatment of supracondylar humer- 
al fractures in children. J Bone Joint Surg Am. 2001;83A3:323-7.

7. Iyengar SR, Hoffinger SA, Townsend DR. Early versus delayed reduction and pinning of type III displaced supracondylar fractures of the humerus in children: a comparative study. J Orthop Trauma. 1999;131:51-5.

8. Omid R, Choi PD, Skaggs DL. Supracondylar humeral fractures in children. J Bone Joint Surg Am. 2008;905:112132.

9. Ramachandran M, Skaggs DL, Crawford HA, Eastwood DM, Lalonde FD, Vitale MG, et al. Delaying treatment of supracondylar fractures in children: has the pendulum swung too far? J Bone Joint Surg Br. 2008;909:1228-33.

10. Walmsley PJ, Kelly MB, Robb JE, Annan IH, Porter DE. Delay increases the need for open reduction of typeIII supracondylar fractures of the humerus. $J$ Bone Joint Surg Br. 2006;884:528-30.

11. Long C, Titus Ngwa Tagang E, Popat RA, Lawong EK, Brown JA, Wren SM. Factors associated with delays to surgical presentation in North-West Cameroon. Surgery. 2015;1583:756-63.

12. Sibinski M, Sharma H, Bennet GC. Early versus delayed treatment of extension type-3 supracondylar fractures of the humerus in children. J Bone Joint Surg Br. 2006;883:380-1.
13. Silva M, Wong TC, Bernthal NM. Outcomes of reduction more than 7 days after injury in supracondylar humeral fractures in children. J Pediatr Orthop.317:751-6. 14. Soucie JM, Wang C, Forsyth A, Funk S, Denny M, Roach KE, et al. Range of motion measurements: reference values and a database for comparison studies. Haemophilia. 2011;173:500-7.

15. Omololu AB, Alonge TO, Adebisi A. A review of 100 cases of supracondylar fractures in children seen in Ibadan. Afr J Med Med Sci. 2000;292:167-9.

16. Igbigbi PS, Manda K. Epidemiology of humeral fractures in Malawi. Int Orthop. 2004;286:338-41.

17. Gaudeuille A, Douzima PM, Sanze BM, Ndemanga JK, Mandaba JL. [Difficulties in the management of supracondylar fractures of the humerus in children living in Central African Republic]. Med Trop Mars. 1998;583:2736.

18. Guifo ML, Tochie JN, Oumarou BN, Tapouh JRM, Bang AG, Ndoumbe A, et al. Paediatric fractures in a sub-saharan tertiary care center: a cohort analysis of demographic characteristics, clinical presentation, therapeutic patterns and outcomes. Pan Afr Med J. 2017;27:46.

19. Keppler P, Salem K, Schwarting B, Kinzl L. The effectiveness of physiotherapy after operative treatment of supracondylar humeral fractures in children. J Pediatr Orthop. 2005;253:314-6. 